\title{
Constraining new physics from Higgs measurements with Lilith-2
}

\author{
Marius Bertrand, ${ }^{a, b}$ Sabine Kraml, ${ }^{a, *}$ Tran Quang Loc, ${ }^{c}$ Dao Thi Nhung ${ }^{d}$ and Le Duc \\ Ninh $^{d}$ \\ ${ }^{a}$ Laboratoire de Physique Subatomique et de Cosmologie, Université Grenoble-Alpes, CNRS/IN2P3, \\ 53 Avenue des Martyrs, F-38026 Grenoble, France \\ ${ }^{b}$ Faculté des Sciences d'Orsay, Univ. Paris-Saclay, 15 rue Georges Clemenceau, F-91405 Orsay, France \\ ${ }^{c}$ Department of Applied Mathematics \& Theoretical Physics, University of Cambridge, Wilberforce Road, \\ Cambridge CB3 OWA, United Kingdom \\ ${ }^{d}$ Institute for Interdisciplinary Research in Science and Education, ICISE, 55121 Quy Nhon, Vietnam \\ E-mail: gusbertrand@orange.fr, sabine.kraml@lpsc.in2p3.fr, \\ loctranq@gmail.com, dtnhung@ifirse.icise.vn, ldninh@ifirse.icise.vn
}

Lilith is a public Python library for constraining new physics from Higgs signal strength measurements. Version 2.0 of Lilith comes with an extensive XML database which includes the ATLAS and CMS Run 2 Higgs results for 36/fb, in addition the the Run 1 results. Both the code and the database were extended from the ordinary Gaussian approximation employed in Lilith-1.1 to using variable Gaussian and Poisson likelihoods. Moreover, Lilith-2 can make use of correlation matrices of arbitrary dimension. We will report on these novelties and ongoing developments. The importance of how correlations and uncertainties are treated will be demonstrated by means of detailed validations of the implemented experimental results. Moreover, we show the effects for global fits of reduced Higgs couplings, 2HDMs of Type I and Type II, and invisible Higgs decays. The program is publicly available on GitHub and can be used to constrain a wide class of new physics scenarios.

Tools for High Energy Physics and Cosmology - TOOLS2020

2-6 November, 2020

Institut de Physique des 2 Infinis (IP2I), Lyon, France

\footnotetext{
${ }^{*}$ Speaker
} 


\section{Introduction}

The LHC runs in 2010-2012 and 2015-2018 have led to a wealth of experimental results on the $125 \mathrm{GeV}$ Higgs boson. From this emerges an increasingly precise picture of the various Higgs production and decay processes, and consequently of the Higgs couplings to the other particles of the Standard Model (SM), notably gauge bosons and third generation fermions. With all measurements so far agreeing with SM predictions, this poses severe constraints on scenarios of new physics, in which the properties of the observed Higgs boson could be affected in a variety of ways. Indeed, beyond-the-SM (BSM) theories typically predict deviations from the SM expectations, due to mixing effects in enlarged Higgs sectors, loop contributions from new particles (or higher-order effects in effective field theories), new decay modes, and so on. The Higgs data have therefore become a major guideline for BSM theories.

Assessing the compatibility of a non-SM Higgs sector with the ATLAS and CMS results requires to construct a likelihood, which is a non-trivial task. While this is best done by the experimental collaborations themselves, having at least an approximate global likelihood is very useful, as it allows theorists to pursue in-depth studies of the implications for their models. For this reason, the public code Lilith [1-3] was created, making use of the Higgs signal strength measurements published by ATLAS and CMS, and the Tevatron experiments. Lilith is a lightweight Python library that uses as a primary input signal strength results

$$
\mu(X, Y) \equiv \frac{\sigma(X) \mathrm{BR}(H \rightarrow Y)}{\sigma^{\mathrm{SM}}(X) \mathrm{BR}^{\mathrm{SM}}(H \rightarrow Y)},
$$

in which the fundamental production and decay modes are unfolded from experimental categories. Here, the main production mechanisms $X$ are: gluon fusion ( $\mathrm{ggH})$, vector-boson fusion (VBF), associated production with an electroweak gauge boson ( $\mathrm{WH}$ and $\mathrm{ZH}$, collectively denoted as $\mathrm{VH}$ ) and associated production with top quarks, mainly $\mathrm{ttH}$ but also tH. The main decay modes $Y$ accessible at the LHC are $H \rightarrow \gamma \gamma, H \rightarrow Z Z^{*} \rightarrow 4 \ell$ and $H \rightarrow W W^{*} \rightarrow 2 \ell 2 v$ (with $\ell \equiv e, \mu$ ), $H \rightarrow b \bar{b}, H \rightarrow \tau^{+} \tau^{-}$and recently also $H \rightarrow \mu^{+} \mu^{-}$.

Version 2 of Lilith [3] features a better treatment of asymmetric uncertainties through the use of variable Gaussian and Poisson likelihoods (as compared to the normal Gaussian approximation in version 1). Moreover, Lilith-2 can make use of correlation matrices of arbitrary dimension. The experimental results used are stored in an easily extendible XML database. The current official release comes with database DB 19.09, which includes the full set of published ATLAS and CMS Run 2 Higgs results for $36 \mathrm{fb}^{-1}$ as of September 2019. An update to the available full Run 2 luminosity $\left(\approx 140 \mathrm{fb}^{-1}\right)$ results is in progress.

\section{The signal strength framework}

The signal strength framework is based on the narrow-width approximation and on the assumption that new physics results only in the scaling of SM Higgs processes. The likelihood in terms of $\mu(X, Y)$, Eq. (1), allows for reinterpretation of the results within models where the signal acceptances for the $(X, Y)$ are to good approximation the same as in the SM; this applies to models which i) have the same tensor structure as the SM and ii) have no new production modes; see e.g. the 
discussion in [4]. This framework can be used to constrain a wide variety of new physics models, see for example [5] and references therein.

Results given in terms of signal strengths can be matched to new physics scenarios with the introduction of factors $\boldsymbol{C}_{X}$ and $\boldsymbol{C}_{Y}$ that scale the amplitudes for the production and decay of the SM Higgs boson, respectively, as

$$
\mu(X, Y)=\frac{\boldsymbol{C}_{X}^{2} \boldsymbol{C}_{Y}^{2}}{\sum_{Y} \boldsymbol{C}_{Y}^{2} \mathrm{BR}^{\mathrm{SM}}(H \rightarrow Y)}
$$

for the different production modes $X \in(\mathrm{ggH}, \mathrm{VBF}, \mathrm{WH}, \mathrm{ZH}, \mathrm{ttH}, \ldots)$ and decay modes $Y \in(\gamma \gamma$, $\left.Z Z^{*}, W W^{*}, b \bar{b}, \tau \tau, \ldots\right)$, where the sum runs over all decays that exist for the SM Higgs boson. The factors $\boldsymbol{C}_{X}$ and $\boldsymbol{C}_{Y}$ can be identified to (or derived from) reduced couplings appearing in an effective Lagrangian. ${ }^{1}$ Following $[5,6]$ and subsequent publications, we employ the notation

$$
\mathcal{L}=g\left[C_{W} m_{W} W^{\mu} W_{\mu}+C_{Z} \frac{m_{Z}}{\cos \theta_{W}} Z^{\mu} Z_{\mu}-\sum_{f} C_{f} \frac{m_{f}}{2 m_{W}} f \bar{f}\right] H,
$$

where $C_{W, Z}$ and $C_{f}(f=t, b, c, \tau, \mu)$ are bosonic and fermionic reduced couplings, respectively. In the limit where all reduced couplings go to 1 , the SM case is recovered. In addition to these tree-level couplings, we define the loop-induced couplings $C_{g}$ and $C_{\gamma}$ of the Higgs to gluons and photons, respectively. If no new particles appear in the loops, $C_{g}$ and $C_{\gamma}$ are computed from the couplings in Eq. (3) following the procedure established in [7]. Alternatively, $C_{g}$ and $C_{\gamma}$ can be taken as free parameters. Apart from the different notation, this is equivalent to the so-called "kappa framework" of [7]. Finally note that often a subset of the $C$ 's in Eq. (3) is taken as universal, in particular $C_{V} \equiv C_{W}=C_{Z}$ (custodial symmetry), $C_{U} \equiv C_{t}=C_{c}$ and $C_{D} \equiv C_{b}=C_{\tau}=C_{\mu}$ like in the Two-Higgs-doublet model (2HDM) of Type II, or $C_{F} \equiv C_{U}=C_{D}$ as in the 2HDM of Type I.

Last but not least, while the signal strength framework in principle requires that the Higgs signal be a sum of processes that exist for the SM Higgs boson, decays into invisible or undetected new particles, affecting only the Higgs total width, can be accounted for through

$$
\mu(X, Y) \rightarrow[1-\mathrm{BR}(H \rightarrow \text { inv. })-\mathrm{BR}(H \rightarrow \text { undet. })] \mu(X, Y)
$$

without spoiling the approximation.

\section{Lilith 2.0 release, database 19.09}

\section{Likelihood approximations}

For a proper inclusion of the recent Run 2 results from ATLAS and CMS, several improvements were necessary in Lilith. First of all, the ordinary Gaussian approximation was no longer sufficient to model the likelihood. We have therefore extended the parametrisation of the likelihood to Gaussian functions of variable width ("variable Gaussian") as well as generalised Poisson functions, following the prescriptions of [8]. Moreover, Lilith can now make use of correlation matrices of arbitrary dimension (for Gaussian likelihoods; Poisson likelihoods are still limited to 2 dimensions).

\footnotetext{
${ }^{1}$ See, e.g., [2] for detailed explanations.
} 


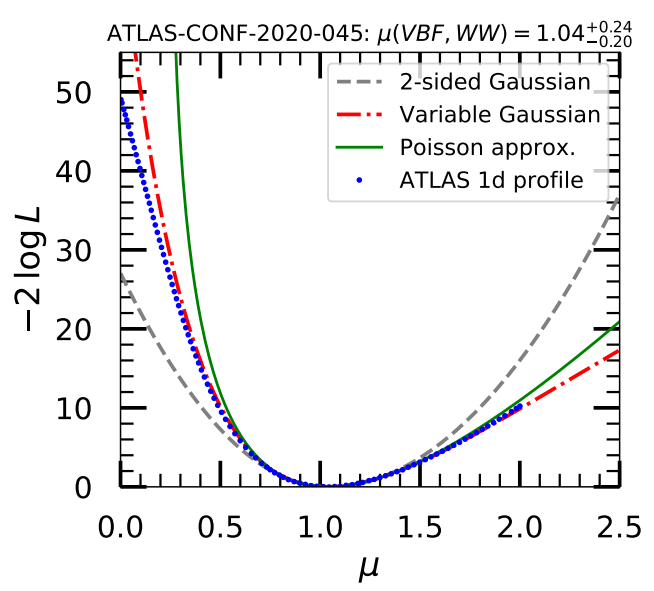

Figure 1: Negative log-likelihoods constructed from $\mu(\mathrm{VBF}, W W)=1.04_{-0.20}^{+0.24}$ [9] using 2-sided Gaussian (grey dashed), variable Gaussian (red dash-dot) and Poisson (green solid) approximations. For comparison, the 1D profile likelihood determined by ATLAS is shown as blue dotted line.

We have also added the tH and the gluon-initiated $\mathrm{ZH}$ production modes, and corrected some minor bugs in the code. The exact way all the above is implemented is described in detail in [3].

The importance of appropriately modelling the shape of the likelihood is illustrated in Figure 1 by means of the ATLAS VBF $\rightarrow H \rightarrow W W^{*} \rightarrow 2 \ell 2 v$ measurement [9]. This ATLAS publication reports $\mu(\mathrm{VBF}, W W)=1.04_{-0.20}^{+0.24}$ as well as a plot of the likelihood profile, which we digitised. As can be seen, the ordinary 2-sided Gaussian, variable Gaussian, and Poisson approximations reproduce the actual likelihood profile, $-2 \log L(\mu)$, with different accuracy. In this example, the best-performing method is the variable Gaussian, but there are other cases where the Poisson distribution gives a better result.

\section{Experimental results in DB19.09}

The current official release, Lilith-2.0, comes with database DB 19.09, which includes the full set of published ATLAS and CMS Run 2 Higgs results for $36 \mathrm{fb}^{-1}$ as of September 2019, summarised in Tables 1 and 2. Each result has been carefully validated. As an example, Figure 2 shows the validation for the CMS combination paper [10] by means of a $C_{F}$ vs. $C_{V}$ fit. Both versions of the implementation use the full $24 \times 24$ correlation matrix given in [10]. However, in the version shown on the left, the plus and minus uncertainties for the 24 channels are described by ordinary Gaussian functions with symmetrised errors, while on the right they are described by Gaussians of variable width. As can be seen, there is a small difference in the resulting errors on $C_{F}$ and $C_{V}$, but most importantly there is a significant shift in the best-fit values. Clearly, the variable Gaussian agrees much better with the official CMS fit, and is hence used in the Lilith database.

In some cases, reproducing the official coupling fits is more tricky. As an illustrative example, we show in Figure 3 fits of $C_{F}$ vs. $C_{V}$ for the data from ATLAS-HIGG-2016-22 ( $\left.H \rightarrow Z Z^{*}\right)$ [11]. The left and middle plots use $\mu\left(\mathrm{ggH}, Z Z^{*}\right)=1.11_{-0.21}^{+0.23}$ and $\mu\left(\mathrm{VBF}, Z Z^{*}\right)=4.0_{-1.46}^{+1.75}$ from Table 9 of [11] with correlation $\rho=-0.41$ from Aux. Fig. $4 \mathrm{c}$ on the analysis twiki page. In the left plot, the likelihood of $\mu\left(\mathrm{ggH}, Z Z^{*}\right)$ vs. $\mu\left(\mathrm{VBF}, Z Z^{*}\right)$ is parametrised as a $2 \mathrm{D}$ variable Gaussian, in the middle plot as a $2 \mathrm{D}$ Poisson distribution. Neither gives a very good agreement with the 


\begin{tabular}{l|ccccccc} 
mode & $\gamma \gamma$ & $Z Z^{*}$ & $W W^{*}$ & $\tau \tau$ & $b \bar{b}$ & $\mu \mu$ & inv. \\
\hline ggH & {$[12]$} & {$[11]$} & {$[13]$} & {$[14]$} & - & {$[15]$} & - \\
$\mathrm{VBF}$ & {$[12]$} & {$[11]$} & {$[13]$} & {$[14]$} & {$[16]$} & {$[15]$} & {$[17]$} \\
$\mathrm{WH}$ & {$[12]$} & {$[11]$} & {$[18]$} & - & {$[19]$} & {$[15]$} & - \\
$\mathrm{ZH}$ & {$[18]$} & - & {$[19]$} & {$[20]$} \\
$\mathrm{ttH}$ & {$[12]$} & {$[11,21]$} & {$[21]$} & {$[21]$} & {$[21,22]$} & - & -
\end{tabular}

Table 1: Overview of ATLAS Run 2 results included in DB 19.09.

\begin{tabular}{l|ccccccc} 
mode & $\gamma \gamma$ & $Z Z^{*}$ & $W W^{*}$ & $\tau \tau$ & $b \bar{b}$ & $\mu \mu$ & inv. \\
\hline $\mathrm{ggH}$ & {$[10]$} & {$[10]$} & {$[10]$} & {$[10]$} & {$[10]$} & {$[10]$} & {$[23]$} \\
$\mathrm{VBF}$ & {$[10]$} & {$[10]$} & {$[10]$} & {$[10]$} & - & {$[10]$} & {$[23]$} \\
$\mathrm{WH}$ & {$[10]$} & {$[10]$} & {$[10]$} & {$[24]$} & {$[10]$} & - & {$[23]$} \\
$\mathrm{ZH}$ & {$[10]$} & {$[10]$} & {$[10]$} & {$[24]$} & {$[10]$} & - & {$[23]$} \\
$\mathrm{ttH}$ & {$[10]$} & {$[10]$} & {$[10]$} & {$[10]$} & {$[10]$} & - & -
\end{tabular}

Table 2: Overview of CMS Run 2 results included in DB 19.09. Note that we use the full $24 \times 24$ correlation matrix for the signal strengths for each production and decay mode combination provided in [10].
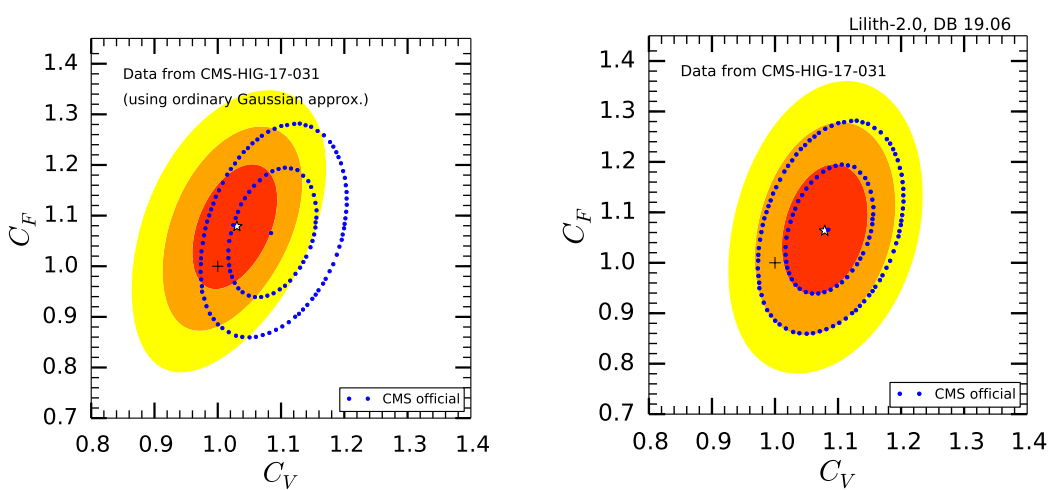

Figure 2: Fit of $C_{F}$ vs. $C_{V}$ using best-fit values and uncertainties for the signal strengths for each production $(\mathrm{ggH}, \mathrm{VBF}, \mathrm{WH}, \mathrm{ZH}, \mathrm{ttH})$ and decay $(\gamma \gamma, \mathrm{ZZ}, W W, \tau \tau, b \bar{b}, \mu \mu)$ mode combination together with the $24 \times 24$ correlation matrix from the CMS combination paper [10]. The $1 \sigma, 2 \sigma$ and $3 \sigma$ regions are shown as red, orange and yellow areas, respectively, and compared to the $1 \sigma$ and $2 \sigma$ contours from CMS (blue dots). On the left, the plus and minus uncertainties are described by ordinary Gaussian functions, while on the right they are described by Gaussians of variable width.

official ATLAS coupling fit. Fortunately, ATLAS also provided 1D profile likelihood plots in their auxiliary material. Fitting these curves as Poisson distributions, we obtain $\mu\left(\mathrm{ggH}, Z Z^{*}\right) \simeq 1.12_{-0.22}^{+0.25}$ and $\mu\left(\mathrm{VBF}, Z Z^{*}\right) \simeq 3.88_{-1.46}^{+1.75}$. With these values, the official ATLAS fit of $C_{F}$ vs. $C_{V}$ can be reproduced well, see the right plot in Figure 3.

\section{Combined coupling fits}

Let us now turn to the question how all these results taken together constrain the Higgs couplings. As an illustrative example, Figure 4 presents combined fits of $C_{F}$ vs. $C_{V}$. The left panel 

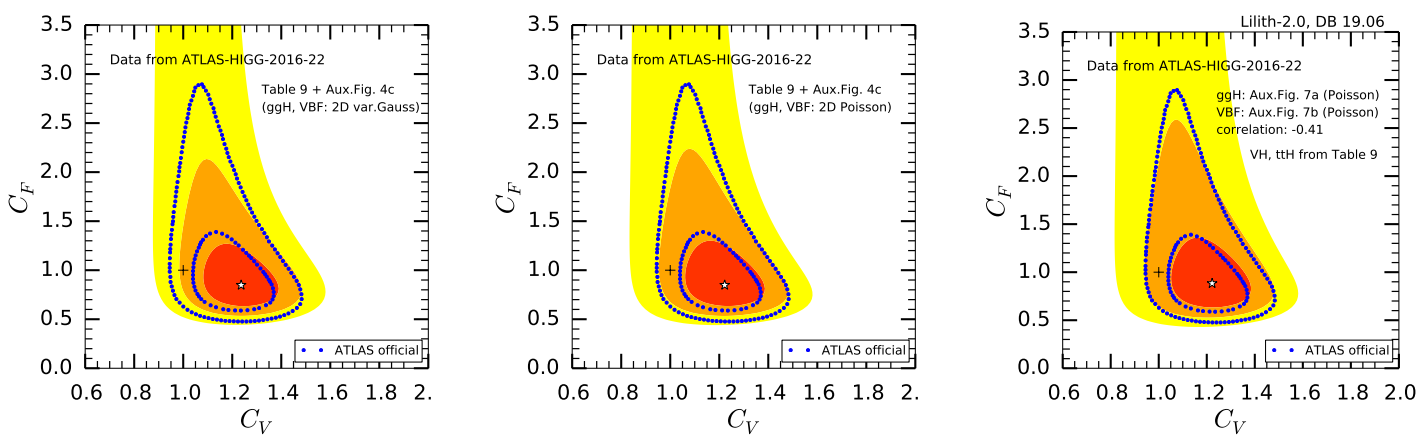

Figure 3: Fit of $C_{F}$ vs. $C_{V}$ for data from the ATLAS $H \rightarrow Z Z^{*}$ analysis, trying different implementations of the $\mu\left(\mathrm{ggH}, Z Z^{*}\right)$ and $\mu\left(\mathrm{VBF}, Z Z^{*}\right)$ values given by ATLAS; see text for details. The $68 \%, 95 \%$ and 99.7\% CL regions obtained with Lilith are shown as red, orange and yellow areas, and compared to the $68 \%$ and $95 \%$ CL contours from ATLAS (in blue). The best-fit point from Lilith is marked as a white star and the $\mathrm{SM}$ as a + .
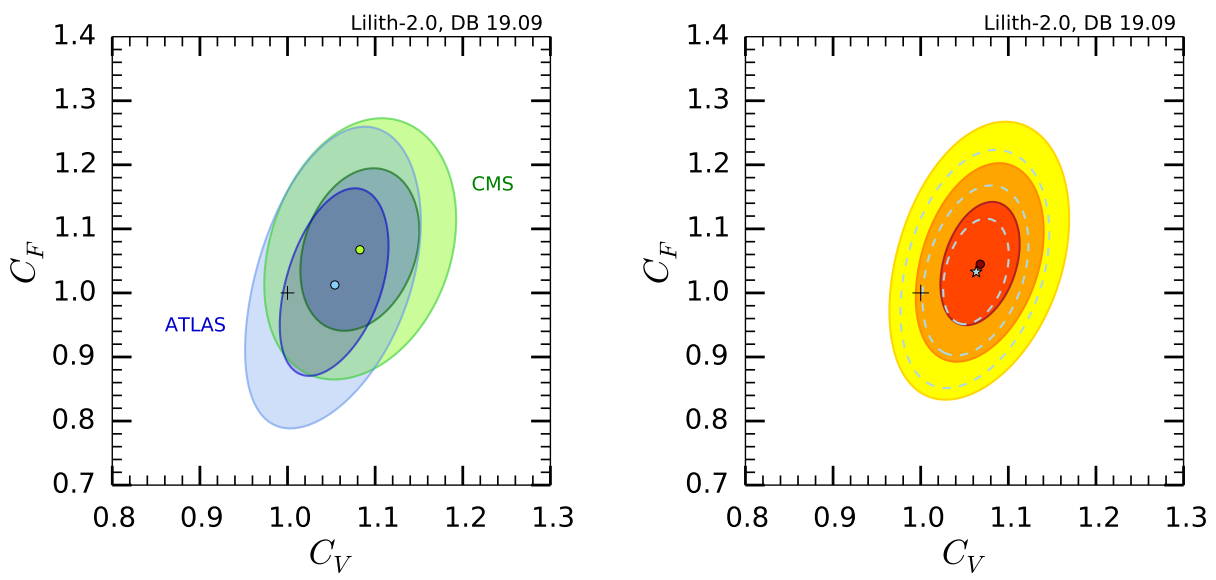

Figure 4: Fits of $C_{F}$ vs. $C_{V}$. Left: $68 \%$ and 95\% CL regions for the Run 2 results from ATLAS (in blue) and from CMS (in green). Right: combination of the ATLAS and CMS Run 2 results in DB 19.09; the $68 \%, 95 \%$ and $99.7 \%$ CL regions are shown as red, orange and yellow areas, respectively. In addition, the light-blue, dashed contours indicate the effect when including also the Run 1 data.

shows the situation when using either the ATLAS (in blue) or the CMS (in green) Run 2 results in DB 19.09. As can be seen, the two experiments agree at the level of about $1 \sigma$, the ATLAS results being slightly closer to the SM (marked as a black + ). The situation when combining the results from both experiments is shown in the right panel.

As an example for a model with extended Higgs sector we consider the 2HDM of Type I and Type II. The couplings of the lighter scalar $h$ are $C_{F}=\cos \alpha / \sin \beta$ in Type I, and $C_{U}=\cos \alpha / \sin \beta$ and $C_{D}=-\sin \alpha / \cos \beta$ in Type II; $C_{V}=\sin (\beta-\alpha)$ in both models. The fit results in the $\tan \beta$ vs. $\cos (\beta-\alpha)$ plane are shown in Figure 5. Note that for Type II the banana-shaped second branch corresponds to the "opposite-sign" solution for the bottom Yukawa coupling [25].

The signal strength measurements in the SM final states also strongly constrain possible invisible (or undetected) Higgs decays, $H \rightarrow$ inv. (or $H \rightarrow$ undet.), as they would reduce the 

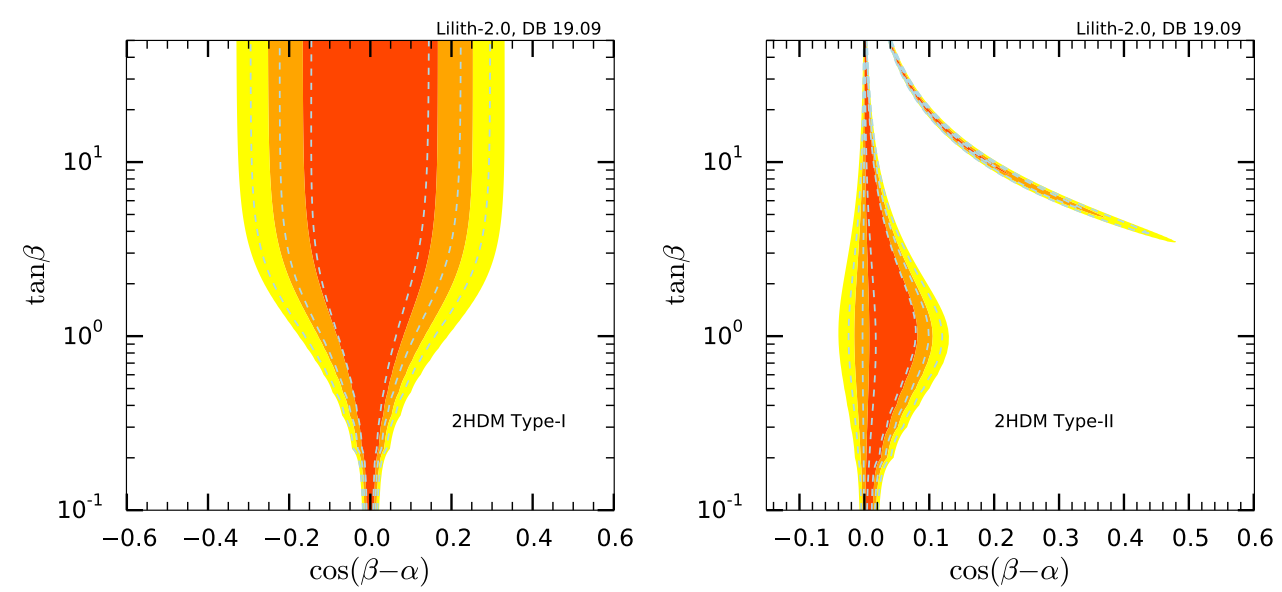

Figure 5: Fits of $\tan \beta$ vs. $\cos (\beta-\alpha)$ for the 2HDM of Type I (left) and of Type II (right) from a combination of the ATLAS and CMS Run 2 results in DB 19.09. The red, orange and yellow areas are the 68\%, 95\% and $99.7 \%$ CL regions, respectively. In addition, the light-blue, dashed contours indicate the $68 \%, 95 \%$ and $99.7 \%$ CL regions when combining the Run 2 and Run 1 data. Loop contributions from charged Higgs bosons are neglected and decays into non-SM particles (such as $h \rightarrow A A$ ) assumed to be absent.

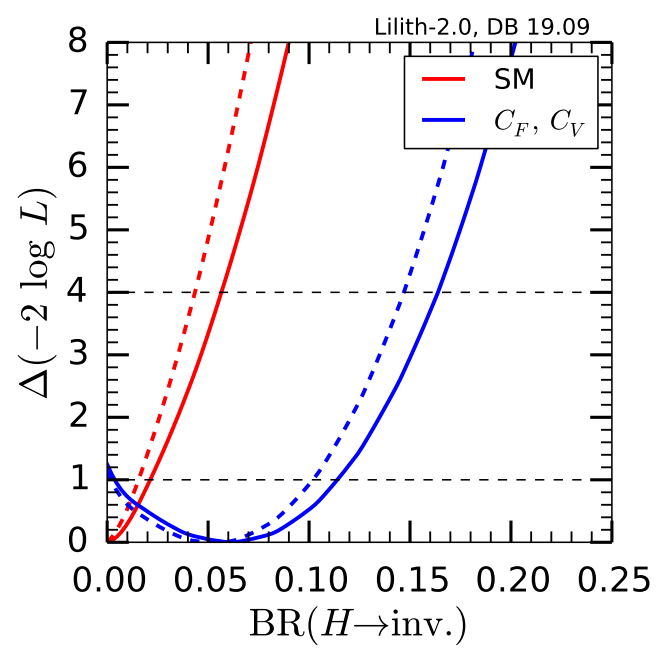

Figure 6: $1 \mathrm{D}$ profile likelihoods of $\mathrm{BR}(H \rightarrow$ inv. $)$, in red for $\mathrm{SM}\left(C_{F}=C_{V}=1\right)$ couplings, in blue for $C_{F}$, $C_{V}$ as free parameters; full lines are for Run 2, while dashed lines are for Run $2+$ Run 1 results in DB 19.09.

rates of (visible) SM final states [26], see Eq. (4). For illustration, Figure 6 shows the 1D profile likelihood of $\mathrm{BR}\left(H \rightarrow\right.$ inv.) for two cases, SM couplings (in red) and $C_{F}$ and $C_{V}$ as free parameters (in blue). We find that the Run 2 (Run 2+Run 1) results in DB 19.09 constrain $\mathrm{BR}(H \rightarrow$ inv.) $\lesssim 5 \%$ $(4 \%)$ at $95 \% \mathrm{CL}$ for the SM-like case, and to $\mathrm{BR}(H \rightarrow$ inv. $) \lesssim 16 \%(15 \%)$ when $C_{F}$ and $C_{V}$ are treated as free parameters; the case of free $C_{U}, C_{D}, C_{V}$ is not shown but gives the same result. These constraints are much stronger than those from the dedicated searches for $H \rightarrow$ inv. decays. 

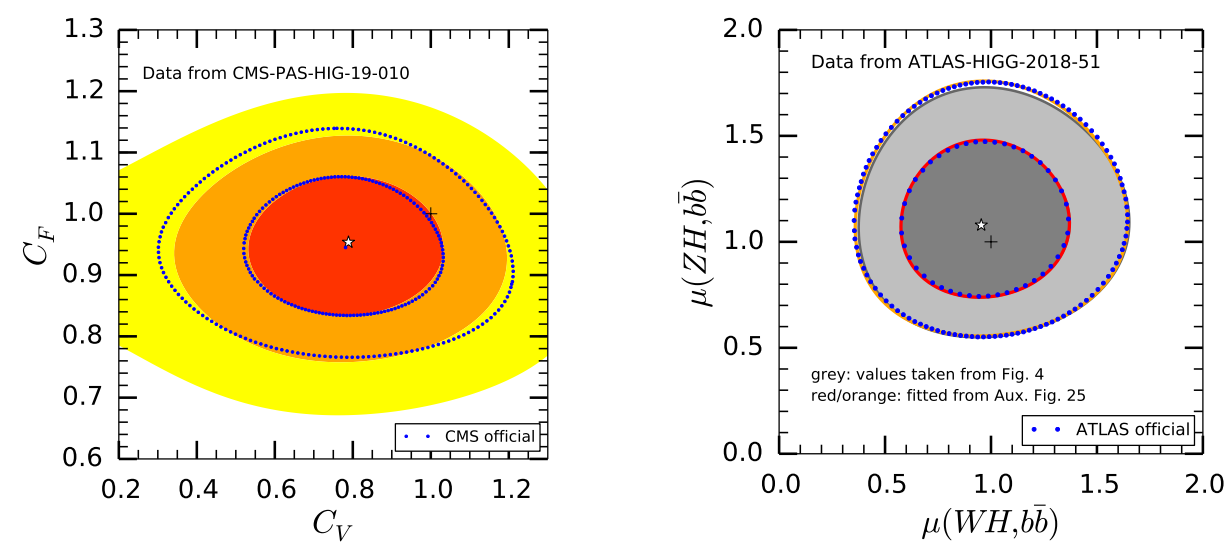

Figure 7: Validation plots for the implementation of the results of the CMS-PAS-HIG-19-010 [27] (left) and ATLAS-HIGG-2018-51 [28] (right) analyses; see text for details.

\section{Ongoing developments and deployment}

Lilith is open source software distributed under the terms of the GNU General Public License. It is available from

https://github.com/sabinekraml/Lilith-2/.

The current official release, v2.0 with DB19.09 (see the releases page) is written in Python 2. A Python 3 version is available as v2.1 pre-release. The usage of the code is explained in [2] and the tutorial session of this workshop.

Ongoing developments concentrate on the extension of the database with the ATLAS and CMS results for full Run 2 luminosity. Some implementations+validations are straightforward, like for the CMS measurement in the $H \rightarrow \tau \tau$ channel, CMS-PAS-HIG-19-010 [27], shown in Figure 7 (left): here, the best-fit values and uncertainties for $\mu(\mathrm{VBF}, \tau \tau)$ and $\mu(\mathrm{ggH}, \tau \tau)$ are taken from Fig. 10 of [27] and their correlation $\rho=-0.265$ fitted from the 95\% CL contour in Fig. 14-b of [27]. The likelihood is modelled as a $2 \mathrm{D}$ variable Gaussian. For validation, we compare to the $68 \%$ and $95 \% \mathrm{CL}$ contours in the $C_{F}$ vs. $C_{V}$ plane shown in Fig. 14-a of the CMS paper and find good agreement.

For other publications, like the ATLAS measurements of $W H$ and $Z H$ production in the $H \rightarrow b \bar{b}$ decay channel, ATLAS-HIGG-2018-51 [28], the possibilities for validation are limited to reproducing contours of constant signal strengths, see Figure 7 (right). ${ }^{2}$ Here, the grey contours show the $68 \%$ and $95 \% \mathrm{CL}$ regions obtained when taking the uncertainties for $\mu(W H, b \bar{b})$ and $\mu(Z H, b \bar{b})$ from Fig. 4 of [28], with correlation $\rho=0.027$ as mentioned in the paper's text. The comparison with the official ATLAS contours from Auxiliary Fig. 25 available on the analysis' twiki page (blue dots) allows to validate the shape of the likelihood, again approximated as a variable Gaussian. The agreement can be improved by fitting the uncertainties on $\mu(W H, b \bar{b})$ and $\mu(Z H, b \bar{b})$ directly from the $95 \%$ CL contour of Aux. Fig. 25, as illustrated by the red and orange contours.

\footnotetext{
${ }^{2}$ The results of this ATLAS paper are available on HEPData, which facilitates a lot their reuse.
} 
There are also a number of new ATLAS and CMS measurements, which do not provide any validation material at all, in which case the (level of accuracy of the) implementations in Lilith cannot be verified. New difficulties also arise from the fact that some ATLAS studies [29, 30] report only simplified template cross sections (STXS) with SM uncertainties factored out, but not any more the per-channel signal strengths including SM uncertainties. The differences in the ATLAS and CMS policies in this respect were discussed by Davide Mungo at the Higgs 2020 conference [31]. We are currently working on the procedure to fold the SM theory uncertainties back in, in order to reproduce the official ATLAS fits with good precision.

\section{Conclusions}

We presented Lilith-2, a light and easy-to-use Python tool for constraining new physics from signal strength measurements of the $125 \mathrm{GeV}$ Higgs boson. The main novelties of v2.0 are:

- a better treatment of asymmetric uncertainties through the use of variable Gaussian or Poisson likelihoods where appropriate;

- the use of multi-dimensional correlations whenever available;

- a well-validated database (DB 19.09) including the published ATLAS and CMS Run 2 Higgs results for $36 \mathrm{fb}^{-1}$.

We discussed the modelling of the likelihood and the implementation and validation of the results in the database. Moreover, we showed examples for combined fits of reduced Higgs couplings, 2HDMs of Type I and Type II, and invisible Higgs decays.

Lilith-2 is publicly available on GitHub and ready to be used to constrain a wide class of new physics scenarios. It is also interfaced from micrOMEGAs [32] (v4.3 or higher) and can thus easily be used for dark matter models - this is particularly useful for, e.g., constraining Higgs decays into dark matter particles in a consistent manner. The code was recently translated from Python 2 to Python 3 in order to meet up-to-date standards. An extension of the database with the available ATLAS and CMS results for full Run 2 luminosity (about $140 \mathrm{fb}^{-1}$ ) is in preparation.

Given the high interest and ease of use of the signal strength framework-indeed a multitude of theory studies are based on Higgs signal strength results-we kindly ask the experimental collaborations to continue to provide detailed $\mu$ values for the various Higgs production $\times$ decay modes, including their correlations. Reference [33] lists detailed recommendations in this respect, to achieve optimal usefulness of the experimental results.

Last but not least we want to note that the publication of full statistical likelihoods, as pioneered in [34] for supersymmetry searches, would be extremely welcome also for Higgs results: it would render the various approximations for modelling the shape of the likelihood (and the associated try-and-error approach in the validation) unnecessary and thus constitute a major leap forward regarding the precision and reliability with which the Higgs results can be reused. 


\section{Acknowledgements}

This work is supported in part by the IN2P3 master project "Théorie - BSMGA". D.T.N. and L.D.N. are funded by the Vietnam National Foundation for Science and Technology Development (NAFOSTED) under grant number 103.01-2020.17.

\section{References}

[1] J. Bernon, B. Dumont and S. Kraml, Status of Higgs couplings after run 1 of the LHC, Phys. Rev. D90 (2014) 071301 [1409.1588].

[2] J. Bernon and B. Dumont, Lilith: a tool for constraining new physics from Higgs measurements, Eur. Phys. J. C75 (2015) 440 [1502.04138].

[3] S. Kraml, T.Q. Loc, D.T. Nhung and L.D. Ninh, Constraining new physics from Higgs measurements with Lilith: update to LHC Run 2 results, SciPost Phys. 7 (2019) 052 [1908.03952].

[4] F. Boudjema et al., On the presentation of the LHC Higgs Results, in Workshop on Likelihoods for the LHC Searches Geneva, Switzerland, January 21-23, 2013, 2013 [1307.5865].

[5] G. Belanger, B. Dumont, U. Ellwanger, J.F. Gunion and S. Kraml, Global fit to Higgs signal strengths and couplings and implications for extended Higgs sectors, Phys. Rev. D88 (2013) 075008 [1306.2941].

[6] G. Belanger, B. Dumont, U. Ellwanger, J.F. Gunion and S. Kraml, Higgs Couplings at the End of 2012, JHEP 02 (2013) 053 [1212. 5244].

[7] LHC Higgs Cross Section Working Group collaboration, Handbook of LHC Higgs Cross Sections: 3. Higgs Properties, 1307.1347.

[8] R. Barlow, Asymmetric statistical errors, in Statistical Problems in Particle Physics, Astrophysics and Cosmology (PHYSTAT 05): Proceedings, Oxford, UK, September 12-15, 2005, pp. 56-59, 2004 [physics/0406120].

[9] ATLAS collaboration, Observation of vector-boson-fusion production of Higgs bosons in the $H \rightarrow W W^{*} \rightarrow e v \mu v$ decay channel in pp collisions at $\sqrt{s}=13 \mathrm{TeV}$ with the ATLAS detector, Tech. Rep. ATLAS-CONF-2020-045, CERN, Geneva (Aug, 2020).

[10] CMS collaboration, Combined measurements of Higgs boson couplings in proton-proton collisions at $\sqrt{s}=13$ TeV, Eur. Phys. J. C79 (2019) 421 [1809. 10733].

[11] ATLAS collaboration, Measurement of the Higgs boson coupling properties in the $H \rightarrow Z Z^{*} \rightarrow 4 \ell$ decay channel at $\sqrt{s}=13 \mathrm{TeV}$ with the ATLAS detector, JHEP 03 (2018) $095[1712.02304]$. 
[12] ATLAS collaboration, Measurements of Higgs boson properties in the diphoton decay channel with $36 \mathrm{fb}^{-1}$ of p collision data at $\sqrt{\mathrm{s}}=13 \mathrm{TeV}$ with the ATLAS detector, Phys. Rev. D98 (2018) 052005 [1802.04146].

[13] ATLAS collaboration, Measurements of gluon-gluon fusion and vector-boson fusion Higgs boson production cross-sections in the $H \rightarrow W W^{*} \rightarrow$ ev $\mu v$ decay channel in pp collisions at $\sqrt{s}=13$ TeV with the ATLAS detector, Phys. Lett. B789 (2019) 508 [1808.09054].

[14] ATLAS collaboration, Cross-section measurements of the Higgs boson decaying into a pair of $\tau$-leptons in proton-proton collisions at $\sqrt{s}=13 \mathrm{TeV}$ with the ATLAS detector, Phys. Rev. D99 (2019) 072001 [1811.08856].

[15] ATLAS collaboration, Search for the dimuon decay of the Higgs boson in pp collisions at $\sqrt{s}=13$ TeV with the ATLAS detector, Phys. Rev. Lett. 119 (2017) 051802 [1705. 04582].

[16] ATLAS collaboration, Search for Higgs bosons produced via vector-boson fusion and decaying into bottom quark pairs in $\sqrt{s}=13 \mathrm{TeV}$ pp collisions with the ATLAS detector, Phys. Rev. D98 (2018) 052003 [1807.08639].

[17] ATLAS collaboration, Combination of searches for invisible Higgs boson decays with the ATLAS experiment, Phys. Rev. Lett. 122 (2019) 231801 [1904.05105].

[18] ATLAS collaboration, Measurement of the production cross section for a Higgs boson in association with a vector boson in the $H \rightarrow W W^{*} \rightarrow \ell v \ell v$ channel in pp collisions at $\sqrt{s}=13 \mathrm{TeV}$ with the ATLAS detector, 1903.10052.

[19] ATLAS collaboration, Evidence for the $H \rightarrow b \bar{b}$ decay with the ATLAS detector, JHEP 12 (2017) 024 [1708.03299].

[20] ATLAS collaboration, Search for an invisibly decaying Higgs boson or dark matter candidates produced in association with a $Z$ boson in pp collisions at $\sqrt{s}=13 \mathrm{TeV}$ with the ATLAS detector, Phys. Lett. B776 (2018) 318 [1708.09624].

[21] ATLAS collaboration, Evidence for the associated production of the Higgs boson and a top quark pair with the ATLAS detector, Phys. Rev. D97 (2018) 072003 [1712 . 08891].

[22] ATLAS collaboration, Search for the standard model Higgs boson produced in association with top quarks and decaying into a b $\bar{b}$ pair in pp collisions at $\sqrt{s}=13 \mathrm{TeV}$ with the ATLAS detector, Phys. Rev. D97 (2018) 072016 [1712. 08895].

[23] CMS collaboration, Search for invisible decays of a Higgs boson produced through vector boson fusion in proton-proton collisions at $\sqrt{s}=13 \mathrm{TeV}, 1809.05937$.

[24] CMS collaboration, Search for the associated production of the Higgs boson and a vector boson in proton-proton collisions at $\sqrt{s}=13 \mathrm{TeV}$ via Higgs boson decays to $\tau$ leptons, JHEP 06 (2019) 093 [1809.03590].

[25] P.M. Ferreira, J.F. Gunion, H.E. Haber and R. Santos, Probing wrong-sign Yukawa couplings at the LHC and a future linear collider, Phys. Rev. D89 (2014) 115003 [1403.4736]. 
[26] G. Belanger, B. Dumont, U. Ellwanger, J.F. Gunion and S. Kraml, Status of invisible Higgs decays, Phys. Lett. B723 (2013) 340 [1302. 5694].

[27] CMS collaboration, Measurement of Higgs boson production in the decay channel with a pair of $\tau$ leptons, Tech. Rep. CMS-PAS-HIG-19-010, CERN, Geneva (2020).

[28] ATLAS collaboration, Measurements of $W H$ and $Z H$ production in the $H \rightarrow b \bar{b}$ decay channel in pp collisions at $13 \mathrm{TeV}$ with the ATLAS detector, 2007.02873.

[29] ATLAS collaboration, Higgs boson production cross-section measurements and their EFT interpretation in the $4 \ell$ decay channel at $\sqrt{s}=13 \mathrm{TeV}$ with the ATLAS detector, Eur. Phys. J. C 80 (2020) 957 [2004.03447].

[30] ATLAS collaboration, A combination of measurements of Higgs boson production and decay using up to $139 \mathrm{fb}^{-1}$ of proton-proton collision data at $\sqrt{\mathrm{s}}=13 \mathrm{TeV}$ collected with the ATLAS experiment, Tech. Rep. ATLAS-CONF-2020-027, CERN, Geneva (Aug, 2020).

[31] D.P. Mungo, Combination of Higgs measurements: $\kappa$ modifiers, STXS framework and MSSM interpretation, Talk given at the Higgs 2020 conference, 26-30 Oct. 2020 (online), https://indico.cern.ch/event/900384/contributions/4059280/.

[32] D. Barducci, G. Belanger, J. Bernon, F. Boudjema, J. Da Silva, S. Kraml et al., Collider limits on new physics within micrOMEGAs_4.3, Comput. Phys. Commun. 222 (2018) 327 [1606.03834].

[33] LHC ReInterpretation Forum, Reinterpretation of LHC Results for New Physics: Status and Recommendations after Run 2, SciPost Phys. 9 (2020) 022 [2003.07868].

[34] ATLAS collaboration, Reproducing searches for new physics with the ATLAS experiment through publication of full statistical likelihoods, Tech. Rep. ATL-PHYS-PUB-2019-029, CERN, Geneva (Aug, 2019). 